\title{
Tailoring the Magnetism of Co Atoms on Graphene through Substrate Hybridization
}

\author{
F. Donati, ${ }^{1}$ L. Gragnaniello, ${ }^{1, *}$ A. Cavallin, ${ }^{1, \dagger}$ F. D. Natterer, ${ }^{1, *}$ Q. Dubout, ${ }^{1}$ M. Pivetta, ${ }^{1}$ F. Patthey, ${ }^{1}$ \\ J. Dreiser, ${ }^{1,2}$ C. Piamonteze, ${ }^{2}$ S. Rusponi, ${ }^{1}$ and H. Brune ${ }^{1}$ \\ ${ }^{1}$ Institute of Condensed Matter Physics (ICMP), École Polytechnique Fédérale de Lausanne (EPFL), \\ Station 3, CH-1015 Lausanne, Switzerland \\ ${ }^{2}$ Swiss Light Source (SLS), Paul Scherrer Institut (PSI), CH-5232 Villigen PSI, Switzerland
}

(Received 9 April 2014; published 20 October 2014)

\begin{abstract}
We determine the magnetic properties of individual Co atoms adsorbed on graphene $(G)$ with $\mathrm{x}$-ray absorption spectroscopy and magnetic circular dichroism. The magnetic ground state of Co adatoms strongly depends on the choice of the metal substrate on which graphene is grown. Cobalt atoms on $\mathrm{G} / \mathrm{Ru}(0001)$ feature exceptionally large orbital and spin moments, as well as an out-of-plane easy axis with large magnetic anisotropy. Conversely, the magnetic moments are strongly reduced for $\mathrm{Co} / \mathrm{G} / \mathrm{Ir}(111)$, and the magnetization is of the easy-plane type. We demonstrate how the Co magnetic properties, which ultimately depend on the degree of hybridization between the Co $3 d$ orbitals and graphene $\pi$ bands, can be tailored through the strength of the graphene-substrate coupling.
\end{abstract}

Graphene (G) recently emerged as one of the most promising materials for spintronics [1]. It was proposed as a perfect spin filter between two ferromagnetic electrodes $[2,3]$, isolated adsorbed magnetic impurities were employed to tailor its electronic [4,5] and spin transport properties [6], and it was used as a capping layer to increase the magnetic anisotropy of ultra thin films [7,8]. In addition, giant magnetic anisotropies were predicted for $3 d$ metal atoms and dimers on graphene $[9,10]$ or on graphene $\mathrm{C}$ vacancies [11], suggesting long spin relaxation times with the potential for quantum information processing in single adatoms.

The magnetic properties of transition metal atoms on graphene strongly depend on the on-site Coulomb interaction [12-14], whose screening is determined by the graphene local density of states around the Fermi level, $E_{F}$ [15]. The latter can be adjusted by the graphenesubstrate hybridization [16] creating the possibility of tailoring the magnetism of transition metal atoms adsorbed onto graphene. Here, we demonstrate this concept by controlling the magnetic properties of Co adatoms up to the point of turning the easy magnetization direction from in plane to out of plane.

We use x-ray absorption spectroscopy (XAS) and x-ray magnetic circular dichroism (XMCD) to reveal the magnetism of ensembles of individual Co atoms adsorbed on graphene grown on $\operatorname{Ru}(0001)$ and $\operatorname{Ir}(111)$. While most of the $\mathrm{G}$ layer hybridizes strongly with $\mathrm{Ru}$ [17], it interacts essentially only by van der Waals forces with Ir [18], as reflected in the very different binding distances of the respective moiré minima of 2.1 versus $3.4 \AA$ [19]. Both metal substrates allow the growth of defect-free monolayer graphene with a filling factor of 1 [20,21], thereby enabling Co adatom ensemble measurements. On G/Ru(0001), we find large Co spin and orbital moments with out-of-plane easy axis and very high magnetic anisotropy. In contrast, Co atoms on $\mathrm{G} / \mathrm{Ir}(111)$ show much lower spin and negligible orbital moments, and they have an out-of-plane hard axis. These findings exemplify the decisive role played by graphene-substrate hybridization on the magnetic properties of single atoms.

We performed XAS and XMCD measurements at the $\mathrm{X}$-Treme beam line of the Swiss Light Source [22]. XAS spectra were recorded with circularly polarized light in the total electron yield (TEY) mode at the Co $L_{2,3}$ absorption edges at $T=2.5 \mathrm{~K}$ and in magnetic fields up to $B=6.8 \mathrm{~T}$ parallel to the $\mathrm{x}$-ray beam. The spectra were normalized to the intensity of the x-ray beam measured on a metallic grid placed upstream of the sample, and in addition to their preedge values at $768.5 \mathrm{eV}$ in order to account for the different TEY efficiencies at normal $\left(0^{\circ}\right)$ and grazing $\left(70^{\circ}\right)$ incidence. The XMCD signal is the difference between the XAS recorded for parallel $\mu_{+}$and antiparallel $\mu_{-}$alignment of the photon helicity with respect to the direction of the applied field. Each pair of $\mu_{+}$and $\mu_{-}$spectra was acquired on a different spot of the sample to prevent beam-induced modifications of the XAS signal [23]. Single crystals of $\mathrm{Ru}(0001)$ and $\operatorname{Ir}(111)$ were prepared in situ by means of $\mathrm{Ar}^{+}$sputtering and annealing $(1400 \mathrm{~K})$ cycles in the preparation chamber of the X-Treme end station. A single layer of graphene was grown by chemical vapor deposition (100 Langmuir $\mathrm{C}_{2} \mathrm{H}_{4}$ at $1400 \mathrm{~K}$ ) and the quality checked with an Omicron VT scanning tunneling microscope (STM) connected to the preparation chamber. Cobalt was deposited from high purity rods $(99.995 \%)$ using a commercial $e$-beam evaporator onto the sample held at about $3.5 \mathrm{~K}$ in the measurement position at a background pressure of $p_{\text {tot }} \leq 5 \times 10^{-11}$ mbar. The Co coverage $(\Theta)$ was calibrated by mapping the covered area with the STM at room 
temperature for close to half-monolayer coverage, and this calibration was used in XAS by evaluating the integrated Co $L_{2,3}$ signal for the same sample. One monolayer (ML) is defined as one Co atom per substrate atom. For samples prepared under identical conditions as those measured with $\mathrm{XMCD}$, we determined the adsorption site, the fraction of Co monomers and dimers in the ensemble, and their local electronic properties with a home-built low temperature (4.7 K) STM. Multiplet calculations were performed using the CTM4XAS55 code [24]. The initial model parameters were taken from literature $[12,13]$ and iteratively refined to reproduce our experimental spectra (see the Supplemental Material [25]).

Figure 1(a) shows the XAS spectra of $0.01 \mathrm{ML}$ of $\mathrm{Co} / \mathrm{G} /$ $\mathrm{Ru}(0001)$ for normal and grazing photon incidence. At this low Co coverage, the $L_{2,3}$ edges appear as small peaks on top of the substrate background. A STM image of 0.008 ML of $\mathrm{Co}$ on $\mathrm{G} / \mathrm{Ru}(0001)$ is shown in Fig. 1(b). The $(23 \times 23)$ moiré $[42,43]$ is imaged as four different hills per unit cell $[17,44]$. Following the statistical analysis outlined in Refs. [45,46] and in the Supplemental Material [25], we find that the majority of the protrusions are single Co adatoms $(93.0 \pm 0.4 \%)$, the remaining species are dimers. In addition, we verified that hydrogen coadsorption is negligible [46,47]. The XAS signal is also quite different from that of an ensemble of small clusters (see the Supplemental Material [25]). To a very good approximation, therefore, it stems from an ensemble of clean and isolated Co monomers. Moreover, the fine multiplet structure observed in the XAS and XMCD spectra indicates that a large part of the atoms of this ensemble have a very similar electronic structure. Our STM measurements reveal that the majority $(81 \pm 2 \%)$ of the Co atoms are adsorbed in the moiré valleys that make up $2 / 3$ of the surface structure [48]. These atoms are featureless in scanning tunneling spectroscopy in the range of $\pm 40 \mathrm{meV}$ around $E_{F}$. The remaining $19 \pm 2 \%$ of the Co atoms are situated close to the moiré hills and exhibit characteristic Kondo features in their differential conductance [25,49]. Effects of this minority species on the measured XAS signal will be discussed further below. (a)

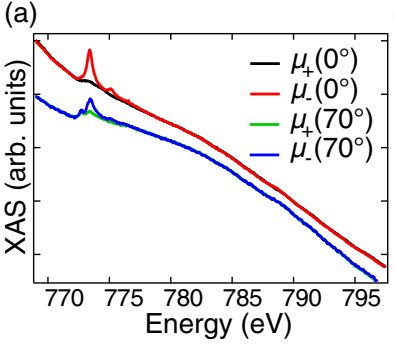

(b)

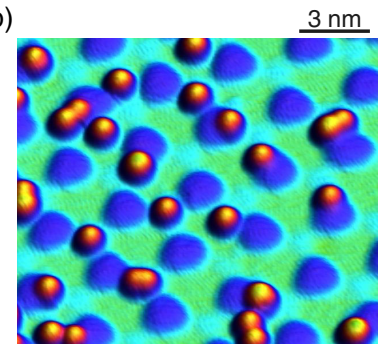

FIG. 1 (color online). (a) Normalized XAS spectra of Co atoms on $\mathrm{G} / \mathrm{Ru}(0001)$ taken at normal and grazing incidence $(\Theta=0.01 \mathrm{ML}$, $B=6.8 \mathrm{~T}, T=2.5 \mathrm{~K}$ ). The spectra are offset for clarity. (b) STM image of $0.008 \mathrm{ML} \mathrm{Co}$ on $\mathrm{G} / \mathrm{Ru}(0001$ ) (mean cluster size $1.07 \pm 0.04$ atoms, $\left.V_{t}=-50 \mathrm{mV}, I_{t}=100 \mathrm{pA}, T=4.7 \mathrm{~K}\right)$.
The magnetic properties and the electronic configuration of the Co adatoms emerge from XAS spectra after subtracting the substrate signal and absorption edge step functions, see Figs. 2(a) and 2(b). A fine multipeak structure is visible for both normal and grazing incidence. In contrast to $\mathrm{Co} / \mathrm{G} / \mathrm{SiC}$ [50] and to $\mathrm{Co}$ on bare $\mathrm{Ru}(0001$ ) (see the Supplemental Material [25]), we find an exceptionally strong dichroism for both incidence geometries, indicating large magnetic moments localized on the Co adatoms. Moreover, the remarkable difference between normal and grazing incidence XMCD reveals a strong uniaxial magnetic anisotropy with an out-of-plane easy axis.

Insight into the electronic structure and the crystal field acting on the Co atoms is obtained from multiplet calculations. As evidenced in Fig. 2(a), the calculated spectra agree very well with the experiment. They reveal a mixed $d^{7}+d^{8}$ configuration with a number of $d$ holes $\left(n_{h}\right)$ of about 2.5. The calculations, further, show that the degeneracy of the $d$ orbitals is lifted by an almost purely uniaxial crystal field, producing an $a_{1}$ singlet $\left(d_{z^{2}}\right)$ and two doublets $e_{1}\left(d_{x z}, d_{y z}\right)$ and $e_{2}\left(d_{x^{2}-y^{2}}, d_{x y}\right)$, respectively. The corresponding splitting of $d$ orbitals is shown in Fig. 2(c) and resembles closely the prediction for a Co atom adsorbed on the graphene top site [12]. The $a_{1}$ singlet is lowest in energy, pointing towards the formation of an out-of-plane bond responsible for the high perpendicular anisotropy (see the Supplemental Material [25]).

Quantitative values of the orbital $\left(m_{L}\right)$ and effective spin magnetic moment (given by the sum of the spin and dipolar

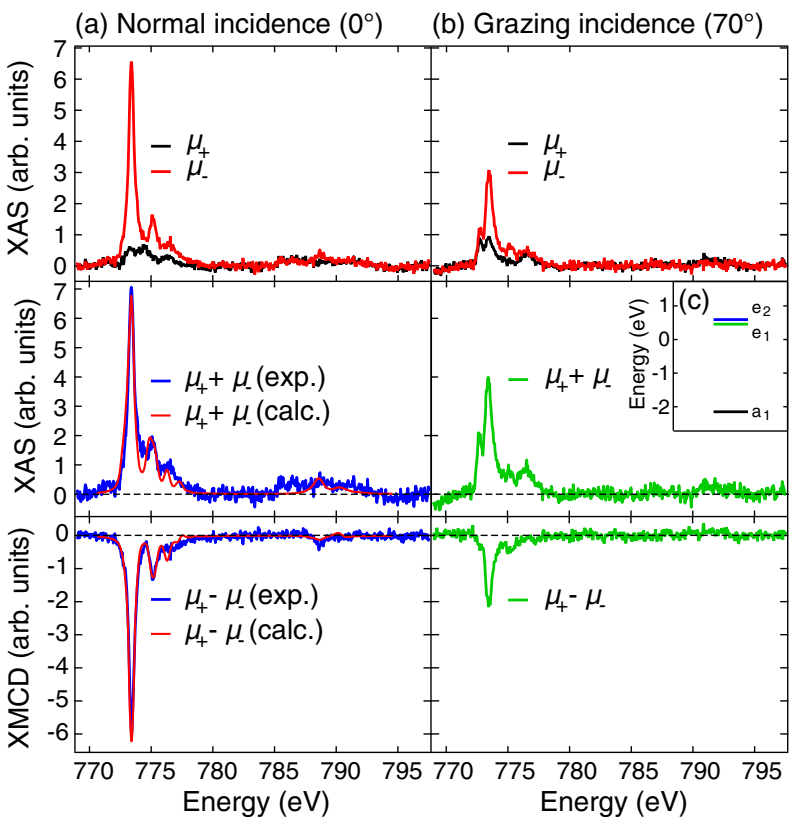

FIG. 2 (color online). (a) and (b) Background subtracted XAS and $\mathrm{XMCD}$ of $\mathrm{Co} / \mathrm{G} / \mathrm{Ru}(0001)$, together with normal incidence spectra from the multiplet calculations $[\Theta=0.01 \mathrm{ML}$, $B=6.8 \mathrm{~T}, T=2.5 \mathrm{~K}$, average over 10 (a), respectively, 5 (b) spectra]. (c) Splitting of $d$ orbitals induced by the uniaxial crystal field according to multiplet calculations. 
TABLE I. Effective spin and orbital magnetic moments (in $\mu_{B}$ ), as well as their sum and ratio, of $\mathrm{Co} / \mathrm{G} / \mathrm{Ru}(0001)$ for normal $\left(0^{\circ}\right)$ and grazing $\left(70^{\circ}\right)$ incidence obtained from experiment (Exp.) using the sum rules with $n_{h}=2.5$. For normal incidence, values corrected (Corr.) for the Kondo screened species and results from multiplet calculations (Mult.) are reported for comparison.

\begin{tabular}{lcccc}
\hline \hline & Exp. $\left(0^{\circ}\right)$ & Corr. $\left(0^{\circ}\right)$ & Mult. $\left(0^{\circ}\right)$ & Exp. $\left(70^{\circ}\right)$ \\
\hline$m_{S+D}$ & $2.26 \pm 0.22$ & 2.79 & 2.95 & $1.25 \pm 0.13$ \\
$m_{L}$ & $1.71 \pm 0.16$ & 2.11 & 2.15 & $0.54 \pm 0.06$ \\
$m_{S+D}+m_{L}$ & $3.97 \pm 0.27$ & 4.90 & 5.10 & $1.79 \pm 0.14$ \\
$m_{L} / m_{S+D}$ & $0.76 \pm 0.14$ & 0.76 & 0.73 & $0.43 \pm 0.09$ \\
\hline \hline
\end{tabular}

terms, $m_{S+D}$ ) projected onto the x-ray incidence direction can be obtained from the integrated XAS and XMCD by application of the sum rules [51,52]. As described in Ref. [53], one needs to account for the strong angular variation of the XAS intensity (see the Supplemental Material [25]). For the individual moments, we used $n_{h}=$ 2.5 from the multiplet calculations. The ratio $m_{L} / m_{S+D}$ is obtained without assumptions on $n_{h}$ and from XMCD alone, which makes it very robust with respect to background subtraction. The resulting magnetic moments, as well as their ratios, are given in Table I. For comparison, we also show normal incidence values obtained from multiplet calculations. The out-of-plane $m_{L}$ value is one of the largest so far reported for magnetic adatoms [54-56]. Also, the anisotropy of $m_{L}$ is exceptionally high, as emphasized by the strong angular variation of the ratio $m_{L} / m_{S+D}$ [57]. Multiplet calculations show excellent agreement with the value of $m_{L} / m_{S+D}$ at normal incidence obtained from the sum rules, but overestimate $m_{S+D}$ and $m_{L}$ by $25 \%-30 \%$. We attribute this to the fraction of Co atoms whose magnetic moments are Kondo screened, thus, giving little or no dichroic contribution [58]. Assuming full screening of the magnetic moment, we corrected for the Kondo species and obtained the $m_{S+D}$ and $m_{L}$ values of the majority population, which agrees very well with those deduced from the multiplet calculations, see Table I.

The magnetic anisotropy $K$ per atom has been quantified by fitting the normal and grazing incidence magnetization curves shown in Fig. 3. In the paramagnetic limit, the expectation value of the Co magnetization can be modeled as [55]

$$
M=M_{\mathrm{sat}} \frac{\int_{0}^{2 \pi} d \phi \int_{0}^{\pi} \sin \theta \cos \theta e^{E / k_{B} T} d \theta}{\int_{0}^{2 \pi} d \phi \int_{0}^{\pi} \sin \theta e^{E / k_{B} T} d \theta},
$$

where $M_{\text {sat }}$ is the saturation magnetization, $\phi$ and $\theta$ are the polar and azimuthal angles of the total magnetic moment $m$ with respect to the surface normal, the energy $E$ is the sum of the Zeeman term $m B \cos \theta$ and the magnetic anisotropy term $-K\left(\sin \theta_{0} \sin \theta \cos \phi+\cos \theta_{0} \cos \theta\right)^{2}$ at a photon incidence angle $\theta_{0}$. From the fit, we obtain $m=5.2 \pm 1.0 \mu_{B}$ and $K=8.4 \pm 2.9 \mathrm{meV}$. We note that a quantum

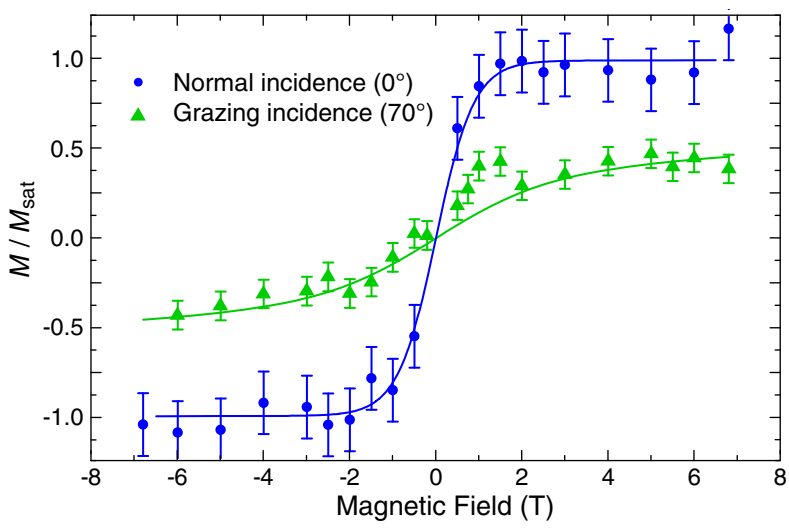

FIG. 3 (color online). Magnetization curve of $\mathrm{Co} / \mathrm{G} / \mathrm{Ru}(0001)$ $(\Theta=0.01 \mathrm{ML}, T=2.5 \mathrm{~K})$. The points correspond to the maximum of the XMCD obtained from a single pair of $\mu_{+}$and $\mu_{-}$ spectra. Since each of them is measured on a different surface spot, we accounted for intensity variations by dividing by the maximum of the background subtracted XAS at the Co $L_{3}$ edge. The XMCD maxima are proportional to the total moments of a Co atom, but are less accurate than the full sum rule analysis taking into account integrals over both $L_{2,3}$ edges. Therefore, we scaled the normal and grazing curves such that their ratio at $6.8 \mathrm{~T}$ is equal to the ratio of the corresponding total moments from sum rules, see Table I. The solid lines show the fits with Eq. (1).

mechanical model based on an effective spin Hamiltonian yields $m$ and $K$ values that agree within the error bars (see the Supplemental Material [25]).

For $3 d$ transition metals with $n_{h}<5$, spin and orbital moments have parallel alignment; therefore, the total atomic moment is $m=m_{S}^{d}+m_{S}^{s}+m_{L}$, where $m_{S}^{d}$ and $m_{S}^{s}$ denote the spin moments due to $d$ and $s$ orbitals, respectively. In order to circumvent the XMCD sensitivity issues for $s$ orbitals [59], we use the value of $0.5 \mu_{B}$ for $m_{S}^{s}$ predicted by density functional theory (DFT) calculations [12]. Taking the corrected values of $m_{S}^{d} \approx m_{S+D}$ [57] and $m_{L}$ from Table I, we obtain $5.4 \pm 0.3 \mu_{B}$, in excellent agreement with the value obtained from the magnetization curves.

The remarkably large anisotropy $K$ approaches the largest values measured for transition metal adatoms on metal and on graphene [47,55], once more illustrating the great potential of graphene to induce large uniaxial magnetic anisotropy. The observation of an out-of-plane easy axis in $\mathrm{Co} / \mathrm{G} / \mathrm{Ru}(0001)$ as opposed to $\mathrm{Co} / \mathrm{G} / \mathrm{Pt}(111)$ [47], both with high anisotropy, is the first evidence of control of the magnetic properties by substrate hybridization.

To further illustrate this concept, we now turn to our second system $\mathrm{Co} / \mathrm{G} / \mathrm{Ir}(111)$, displaying very different magnetic properties and electronic configuration of the adatoms. Figure 4 shows the absence of a fine multipeak structure in XAS that is indicative of a stronger hybridization between Co and graphene orbitals. The $L_{3}$ and $L_{2}$ peaks have opposite signs in the XMCD spectrum resulting from a strongly reduced orbital moment compared to the preceding system. The XMCD signal is largest at grazing 
(a) Normal incidence $\left(0^{\circ}\right)$

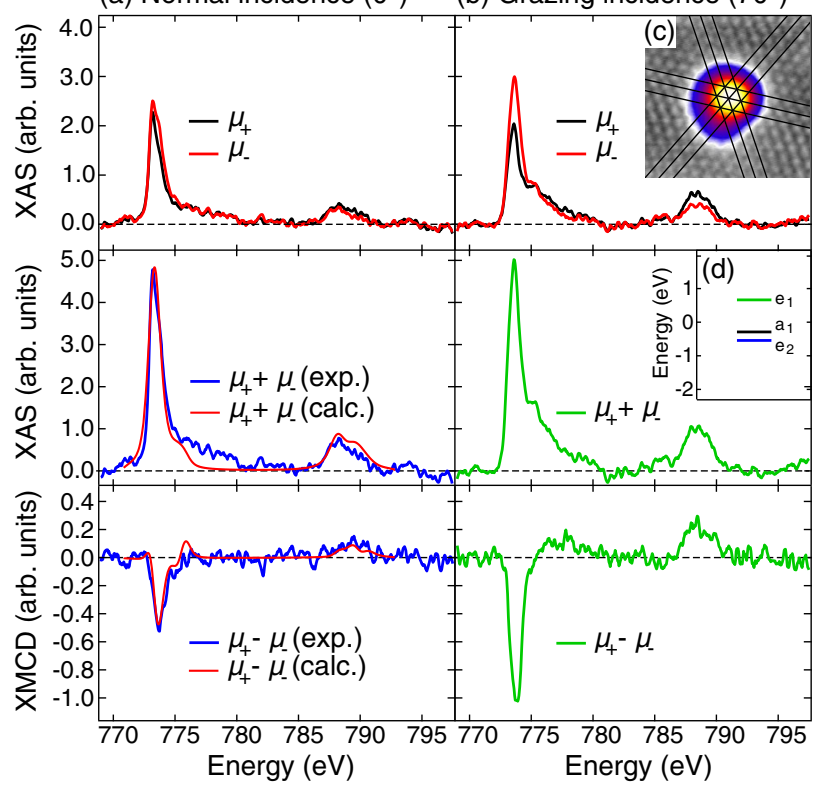

FIG. 4 (color online). (a) and (b) Background subtracted XAS and $\mathrm{XMCD}$ of $\mathrm{Co} / \mathrm{G} / \mathrm{Ir}(111)$, together with normal incidence spectra simulated with multiplet calculations $(\Theta=0.01 \mathrm{ML}$, $B=6.8 \mathrm{~T}, T=2.5 \mathrm{~K}$, (a) and (b) average over six spectra). (c) Adsorption site deduced from STM image of a Co atom on $\mathrm{G} / \mathrm{Ir}(111)$ with atomically resolved graphene. Black lines mark the sixfold graphene hollow sites imaged as protrusions $\left(3.2 \times 3.2 \mathrm{~nm}^{2}, \quad V_{t}=-50 \mathrm{mV}, \quad I_{t}=200 \mathrm{pA}, \quad T=4.7 \mathrm{~K}\right)$. (d) Splitting of $d$ orbitals induced by the uniaxial crystal field according to multiplet calculations.

incidence, revealing an out-of-plane hard axis. Multiplet calculations show an almost pure $d^{8}$ configuration with $n_{h} \approx 1.85$, i.e., $S \approx 1$ (see the Supplemental Material [25]). As expected for an $S=1$ system with an out-of-plane hard axis, neither the in-plane nor the out-of-plane magnetization curves (not shown) saturate at $B=6.8 \mathrm{~T}$ and $T=$ $2.5 \mathrm{~K}$ [60]. Therefore, the atomic magnetic moments obtained from the sum rules given in Table II represent lower bounds to the real values. The atomically resolved STM image shown in Fig. 4(c) reveals that Co adsorbs on the sixfold graphene hollow site. The level splitting of the $d$ orbitals shown in Fig. 4(d) agrees very well with the one predicted by DFT for this adsorption site [12].

Putting the present results into relation with the magnetic properties found for $\mathrm{Co} / \mathrm{G} / \mathrm{Pt}(111)$ [47], we derive a clear correlation between the number of holes in the Co $3 d$ orbitals and the strength of the graphene-substrate interaction which is stronger for $4 d$ and weaker for $5 d$ metals [61]. The number of holes increases from 1.85 for $\mathrm{G} / \mathrm{Ir}(111)$ via 2.0 for $\mathrm{G} / \mathrm{Pt}(111)$ to 2.5 for $\mathrm{G} / \mathrm{Ru}(0001)$, accompanied, as expected, by increasing spin moments. The orbital moments also follow this trend. Similarly, the preferred magnetization direction depends on graphenesubstrate hybridization; it lies in plane for Co on G/Ir(111)
TABLE II. Effective spin and orbital magnetic moments of $\mathrm{Co} / \mathrm{G} / \operatorname{Ir}(111)$, as well as their sum and ratio, estimated from the sum rules using $n_{h}=1.85$.

\begin{tabular}{lcc}
\hline \hline & Normal $\left(0^{\circ}\right)$ & Grazing $\left(70^{\circ}\right)$ \\
\hline$m_{S+D}\left(\mu_{B}\right)$ & $0.45 \pm 0.04$ & $0.57 \pm 0.04$ \\
$m_{L}\left(\mu_{B}\right)$ & $0.09 \pm 0.02$ & $0.04 \pm 0.02$ \\
$m_{S+D}+m_{L}\left(\mu_{B}\right)$ & $0.54 \pm 0.05$ & $0.61 \pm 0.05$ \\
$m_{L} / m_{S+D}$ & $0.19 \pm 0.06$ & $0.07 \pm 0.04$ \\
\hline \hline
\end{tabular}

and on $\mathrm{G} / \mathrm{Pt}(111)$ [47], whereas it is out-of-plane for Co on $\mathrm{G} / \mathrm{Ru}(0001)$.

These findings can be interpreted in the light of the competing hybridization of the graphene $\pi$ bands with both the substrate $d$ bands and Co $3 d$ orbitals. The degree of hybridization of the first is tailored by choosing a specific metal substrate. For $\mathrm{G} / \mathrm{Ru}(0001)$, the strong hybridization with the substrate $d$ bands opens a gap of about $2 \mathrm{eV}$ in the graphene $\pi$ bands just below $E_{F}[62,63]$. This gap limits the hybridization between Co and graphene orbitals and preserves a largely unscreened Coulomb interaction. On the other hand, for $\mathrm{G} / \mathrm{Pt}(111)$ and $\mathrm{G} / \mathrm{Ir}(111)$, the interaction between the substrate and graphene is weak and leaves the graphene $\pi$ bands almost unperturbed [64-66], allowing for a stronger hybridization between Co and graphene and an effective screening of the Coulomb interaction.

Finally, we can correlate the values of the orbital magnetic moment and the change of the easy magnetization direction with the occupation and splitting of the $3 d$ orbitals obtained from multiplet calculations. For $\mathrm{Co} / \mathrm{G} / \mathrm{Ru}(0001)$, the diagonal $e_{1}$ and the planar $e_{2}$ orbitals are highest in energy and almost degenerate; therefore, most of the 2.5 holes are shared between these two doublets. In particular, the hole localized in the $e_{2}$ orbitals preserves a large orbital magnetic moment and favors a perpendicular anisotropy [67]. This behavior is similar to individual Co atoms on $\mathrm{MgO} / \mathrm{Ag}(100)$, where a large unscreened Coulomb interaction, due to the wide $\mathrm{MgO}$ band gap centered at Fermi level, favors fully unquenched orbital moments and maximum magnetic anisotropy [68]. The orbital splitting found for $\mathrm{Co} / \mathrm{G} / \mathrm{Ir}(111)$ is, instead, quite similar to the one calculated for $\mathrm{Co} / \mathrm{G} / \mathrm{Pt}(111)$ [47]. In this case, the two holes are localized in the uppermost $e_{1}$ orbitals. This configuration leads to the quenching of the orbital moment and favors an out-of-plane hard axis.

In conclusion, we have demonstrated how the magnetic properties of Co atoms adsorbed on graphene can be tailored by choosing the appropriate metal substrate. In particular, the complex interplay between the Co $3 d$ orbitals and the graphene $\pi$ bands, modified by the underlying substrate, can generate large magnetic moments and perpendicular magnetic anisotropy in Co atoms, two fundamental requirements for information storage devices and manipulation of single magnetic atoms. 
We acknowledge funding from the Swiss National Science Foundation and from the Sino-Swiss Science and Technology Cooperation Project No. IZLCZ2 123892 .

*Present address: Fachbereich Physik, Universität Konstanz, D-78457 Konstanz, Germany.

${ }^{\dagger}$ Present address: Max-Planck-Institut für Mikrostrukturphysik, D-06120 Halle (Saale), Germany.

*Present address: Center for Nanoscale Science and Technology, NIST, Gaithersburg, MD 20899, USA.

[1] N. Tombros, C. Jozsa, M. Popinciuc, H. T. Jonkman, and B. J. van Wees, Nature (London) 448, 571 (2007).

[2] V. M. Karpan, G. Giovannetti, P. A. Khomyakov, M. Talanana, A. A. Starikov, M. Zwierzycki, J. van den Brink, G. Brocks, and P. J. Kelly, Phys. Rev. Lett. 99, 176602 (2007).

[3] O. V. Yazyev and A. Pasquarello, Phys. Rev. B 80, 035408 (2009).

[4] V. W. Brar, R. Decker, H. M. Solowan, Y. Wang, L. Maserati, K. T. Chan, H. Lee, Ç. O. Girit, A. Zettl, S. G. Louie, M. L. Cohen, and M. F. Crommie, Nat. Phys. 7, 43 (2011).

[5] Y. Wang, V. W. Brar, A. V. Shytov, Q. Wu, W. Regan, H. Z. Tsai, A. Zettl, L. S. Levitov, and M. F. Crommie, Nat. Phys. 8, 653 (2012).

[6] K. Pi, W. Han, K. M. McCreary, A. G. Swartz, Y. Li, and R. K. Kawakami, Phys. Rev. Lett. 104, 187201 (2010).

[7] N. Rougemaille, A. T. N'Diaye, J. Coraux, C. Vo-Van, O. Fruchart, and A. K. Schmid, Appl. Phys. Lett. 101, 142403 (2012).

[8] R. Decker, J. Brede, N. Atodiresei, V. Caciuc, S. Blügel, and R. Wiesendanger, Phys. Rev. B 87, 041403 (2013).

[9] R. J. Xiao, D. Fritsch, M. D. Kuz'min, K. Koepernik, H. Eschrig, M. Richter, K. Vietze, and G. Seifert, Phys. Rev. Lett. 103, 187201 (2009).

[10] M. Sargolzaei and F. Gudarzi, J. Appl. Phys. 110, 064303 (2011).

[11] H. C. Kandpal, K. Koepernik, and M. Richter, Phys. Rev. B 86, 235430 (2012).

[12] T. O. Wehling, A. V. Balatsky, M. I. Katsnelson, A. I. Lichtenstein, and A. Rosch, Phys. Rev. B 81, 115427 (2010).

[13] T. O. Wehling, A. I. Lichtenstein, and M. I. Katsnelson, Phys. Rev. B 84, 235110 (2011).

[14] A. N. Rudenko, F. J. Keil, M. I. Katsnelson, and A. I. Lichtenstein, Phys. Rev. B 86, 075422 (2012).

[15] D. Jacob and G. Kotliar, Phys. Rev. B 82, 085423 (2010).

[16] A. Dahal, R. Addou, H. Coy-Diaz, J. Lallo, and M. Batzill, APL Mat. 1, 042107 (2013).

[17] M. Iannuzzi, I. Kalichava, H. Ma, S. J. Leake, H. Zhou, G. Li, Y. Zhang, O. Bunk, H. Gao, J. Hutter, P. R. Willmott, and T. Greber, Phys. Rev. B 88, 125433 (2013).

[18] C. Busse, P. Lazić, R. Djemour, J. Coraux, T. Gerber, N. Atodiresei, V. Caciuc, R. Brako, A. T. NDiaye, S. Blügel, J. Zegenhagen, and T. Michely, Phys. Rev. Lett. 107, 036101 (2011).

[19] M. Batzill, Surf. Sci. Rep. 67, 83 (2012).

[20] J. Wintterlin and M. L. Bocquet, Surf. Sci. 603, 1841 (2009).
[21] F. D. Natterer, S. Rusponi, M. Papagno, C. Carbone, and H. Brune, J. Phys. Condens. Matter 24, 314203 (2012).

[22] C. Piamonteze, U. Flechsig, S. Rusponi, J. Dreiser, J. Heidler, M. Schmidt, R. Wetter, M. Calvi, T. Schmidt, H. Pruchova, J. Krempasky, C. Quitmann, H. Brune, and F. Nolting, J. Synchrotron Radiat. 19, 661 (2012).

[23] A. Lehnert, S. Rusponi, M. Etzkorn, S. Ouazi, P. Thakur, and H. Brune, Phys. Rev. B 81, 104430 (2010).

[24] E. Stavitski and F. M. F. de Groot, Micron 41, 687 (2010).

[25] See Supplemental Material at http://link.aps.org/ supplemental/10.1103/PhysRevLett.113.177201, which includes Refs. [26-51], for details about STM experiments, multiplet calculations, XAS and XMCD data analysis, as well as for further XAS and XMCD measurements on Co single adatoms on $\operatorname{Ru}(0001)$ and $\operatorname{Ir}(111)$ and small $\mathrm{Co}$ clusters on graphene.

[26] R. Gaisch, J. K. Gimzewski, B. Reihl, R. R. Schlittler, M. Tschudy, and W.-D. Schneider, Ultramicroscopy 42-44, 1621 (1992).

[27] E. Loginova, N. C. Bartelt, P. J. Feibelman, and K. F. McCarty, New J. Phys. 11, 063046 (2009).

[28] P. W. Sutter, J.-I. Flege, and E. A. Sutter, Nat. Mater. 7, 406 (2008).

[29] H. Brune, Surf. Sci. Rep. 31, 125 (1998).

[30] P. Błoński, A. Lehnert, S. Dennler, S. Rusponi, M. Etzkorn, G. Moulas, P. Bencok, P. Gambardella, H. Brune, and J. Hafner, Phys. Rev. B 81, 104426 (2010).

[31] H. Brune, Physics of Covered Solid Surfaces, edited by H. P. Bonzel, Landolt Börnstein New Series, Group III: Condensed Matter Vol. III/42, subvolume A, Part 1 (Springer, Berlin, 2001), p. 217.

[32] M. Gyamfi, T. Eelbo, M. Wasniowska, and R. Wiesendanger, Phys. Rev. B 84, 113403 (2011).

[33] V. Madhavan, W. Chen, T. Jamneala, M. F. Crommie, and N. S. Wingreen, Science 280, 567 (1998).

[34] A. F. Otte, M. Ternes, K. v. Bergmann, S. Loth, H. Brune, C. P. Lutz, C. F. Hirjibehedin, and A. J. Heinrich, Nat. Phys. 4, 847 (2008).

[35] G. E. Pacchioni, M. Pivetta, L. Gragnaniello, F. Donati, S. Rusponi, and H. Brune (unpublished).

[36] A. Abragam and B. Bleaney, Electron Paramagnetic Resonance of Transition Ions (Clarendon, Oxford, 1970).

[37] J. C. Hempel and M. E. Miller, J. Chem. Phys. 75, 2959 (1981).

[38] J. Stöhr and D. A. Outka, Phys. Rev. B 36, 7891 (1987).

[39] C. Brouder, J. Phys. Condens. Matter 2, 701 (1990).

[40] H. Brune and P. Gambardella, Surf. Sci. 603, 1812 (2009).

[41] C. Etz, J. Zabloudil, P. Weinberger, and E. Y. Vedmedenko, Phys. Rev. B 77, 184425 (2008).

[42] D. Martoccia, P. R. Willmott, T. Brugger, M. Björck, S. Günther, C. M. Schlepütz, A. Cervellino, S. A. Pauli, B. D. Patterson, S. Marchini, J. Wintterlin, W. Moritz, and T. Greber, Phys. Rev. Lett. 101, 126102 (2008).

[43] W. Moritz, B. Wang, M.-L. Bocquet, T. Brugger, T. Greber, J. Wintterlin, and S. Günther, Phys. Rev. Lett. 104, 136102 (2010).

[44] Q. Dubout, F. Calleja, G. Sclauzero, M. Etzkorn, A. Lehnert, L. Claude, M. Papagno, F. D. Natterer, F. Patthey, S. Rusponi, A. Pasquarello, and H. Brune (unpublished). 
[45] F. D. Natterer, F. Patthey, and H. Brune, Phys. Rev. Lett. 109, 066101 (2012).

[46] F. D. Natterer, F. Patthey, and H. Brune, Surf. Sci. 615, 80 (2013).

[47] F. Donati, Q. Dubout, G. Autès, F. Patthey, F. Calleja, P. Gambardella, O. V. Yazyev, and H. Brune, Phys. Rev. Lett. 111, 236801 (2013).

[48] The valleys of the moiré correspond to the regions with the $\mathrm{C}-\mathrm{Ru}$ stacking $(\mathrm{C}, \mathrm{C})=($ top, fcc $)$ and $(\mathrm{C}, \mathrm{C})=(\mathrm{hcp}$, top $)$, while the hills correspond to the $(\mathrm{C}, \mathrm{C})=(\mathrm{fcc}, \mathrm{hcp})$.

[49] J. Ren, H. Guo, J. Pan, Y. Y. Zhang, X. Wu, H.-G. Luo, S. Du, S. T. Pantelides, and H.-J. Gao, Nano Lett. 14, 4011 (2014).

[50] T. Eelbo, M. Waśniowska, P. Thakur, M. Gyamfi, B. Sachs, T. O. Wehling, S. Forti, U. Starke, C. Tieg, A. I. Lichtenstein, and R. Wiesendanger, Phys. Rev. Lett. 110, 136804 (2013).

[51] B. T. Thole, P. Carra, F. Sette, and G. van der Laan, Phys. Rev. Lett. 68, 1943 (1992).

[52] P. Carra, B. T. Thole, M. Altarelli, and X. Wang, Phys. Rev. Lett. 70, 694 (1993).

[53] S. Stepanow, A. Mugarza, G. Ceballos, P. Moras, J. C. Cezar, C. Carbone, and P. Gambardella, Phys. Rev. B 82, 014405 (2010).

[54] P. Gambardella, S. S. Dhesi, S. Gardonio, C. Grazioli, P. Ohresser, and C. Carbone, Phys. Rev. Lett. 88, 047202 (2002).

[55] P. Gambardella, S. Rusponi, M. Veronese, S. S. Dhesi, C. Grazioli, A. Dallmeyer, I. Cabria, R. Zeller, P. H. Dederichs, K. Kern, C. Carbone, and H. Brune, Science 300, 1130 (2003).

[56] T. Eelbo, M. Waśniowska, M. Sikora, M. Dobrzański, A. Kozłowski, A. Pulkin, G. Autès, I. Miotkowski, O. V. Yazyev, and R. Wiesendanger, Phys. Rev. B 89, 104424 (2014).
[57] Multiplet calculations reveal a dipolar term $m_{D}=0.45 \mu_{B}$, parallel to $m_{S}$ at normal incidence. Including it, we obtain $m_{L}\left(0^{\circ}\right) / m_{S}\left(0^{\circ}\right)=0.86$, which emphasizes, even more, the large anisotropy of $m_{L}$.

[58] M. Höck and J. Schnack, Phys. Rev. B 87, 184408 (2013).

[59] H. Ebert, J. Stöhr, S. S. P. Parkin, M. Samant, and A. Nilsson, Phys. Rev. B 53, 16067 (1996).

[60] Using Eq. (1) with $m=2 \mu_{B}$ and $K<0$, one observes that $M=0.9 M_{\text {sat }}$ at $B=6.8 \mathrm{~T}$ can be reached only for $T \leq 1 \mathrm{~K}$ along the grazing incidence direction.

[61] A. B. Preobrajenski, M. L. Ng, A. S. Vinogradov, and N. Mårtensson, Phys. Rev. B 78, 073401 (2008).

[62] T. Brugger, S. Günther, B. Wang, H. Dil, M.-L. Bocquet, J. Osterwalder, J. Wintterlin, and T. Greber, Phys. Rev. B 79, 045407 (2009).

[63] D. Stradi, S. Barja, C. Díaz, M. Garnica, B. Borca, J. J. Hinarejos, D. Sánchez-Portal, M. Alcamí, A. Arnau, A. L. Vázquez de Parga, R. Miranda, and F. Martín, Phys. Rev. B 88, 245401 (2013).

[64] P. Sutter, J. T. Sadowski, and E. Sutter, Phys. Rev. B 80, 245411 (2009).

[65] I. Pletikosić, M. Kralj, P. Pervan, R. Brako, J. Coraux, A. T. NDiaye, C. Busse, and T. Michely, Phys. Rev. Lett. 102, 056808 (2009).

[66] S. Rusponi, M. Papagno, P. Moras, S. Vlaic, M. Etzkorn, P. M. Sheverdyaeva, D. Pacilé, H. Brune, and C. Carbone, Phys. Rev. Lett. 105, 246803 (2010).

[67] J. Stöhr and H. C. Siegmann, Magnetism-From Fundamental to Nanoscale Dynamics (Springer, Berlin, 2006).

[68] I. G. Rau, S. Baumann, S. Rusponi, F. Donati, S. Stepanow, L. Gragnaniello, J. Dreiser, C. Piamonteze, F. Nolting, S. Gangopadhyay, O. R. Albertini, R. M. Macfarlane, C. P. Lutz, B. A. Jones, P. Gambardella, A. J. Heinrich, and H. Brune, Science 344, 988 (2014). 